\title{
Pathophysiology of the functional constipation in elderly
}

\author{
MICHAEL D. LEVIN \\ State Geriatric Center, Netanya, Israel
}

\begin{abstract}
The aim of this study was to investigate the pathophysiology of functional constipation (FC) in the elderly. Material and methods: In the State Geriatric Center of Netanya (Israel), 37 patients at the age of 65-93 years with complaints of chronic constipation were examined. This main group did not include patients with tumors and cases after surgery on the rectum and colon. The results of a survey of 15 young people aged 11-17 years with no bowel disease were taken as a control group. A barium enema with a radiopaque marker near the anus was produced. A frontal radiograph of the abdomen as well as an X-ray of the lateral projection of the anorectal zone were made after the administration of 100 , 200 and $300 \mathrm{ml}$ of barium. Results: In $6(16 \%)$ cases, the width of the rectum was more than $5 \mathrm{~cm}-$ megarectum. The length of the anal canal was 2 times less than the minimum normal limit after the injection of $100 \mathrm{ml}$ of barium. In 25 (68\%) patients, the width of the rectum was normal and had a normal shape. The functioning anal canal length was shortened during the administration of barium. In 6 (16\%) patients, the reason for constipation was high colonic tone. They had the anorectal parameters within the normal range. Conclusion: We found 3 types of FC in the elderly. In the structure of FC the cases of self-defined constipation (68\%) are dominated, where there is no mechanical obstruction for defecation. The reason for the sense of constipation and incomplete emptying is due to the age-related weakness of the pelvic floor muscles. In $16 \%$ of patients, obstructive constipation with a megarectum and a fixed descending perineum syndrome was detected. This pathology originated in childhood or after anal fissures, and childbirth. In $16 \%$ of patients, the cause of constipation was a high tone of the colon, what can be the result of an irritable bowel syndrome, the formation of functional sphincters in the colon and diverticula of the sigmoid colon. An increase in the tone of the colon causes a slow transit constipation.
\end{abstract}

Keywords: Elderly; Barium enema; Functional constipation; Outlet obstruction; Slow transit constipation; Anal canal length

\section{INTRODUCTION}

The prevalence of constipation in the general population is approximately $20 \%$ although it can range anywhere from $2 \%$ to $27 \%$, depending on the definition used and population studied. A population-based study reported that the cumulative incidence of chronic constipation is higher in the elderly $(\sim 20 \%)$ compared to a younger population. Severe constipation is more common in elderly women, with rates of constipation two to three times higher than that of their male counterparts ${ }^{1}$. The new criteria for ROME IV represent another attempt by brain noise to streamline scientific research in order to understand the pathophysiology of chronic constipation in patients of different age and sex, which should improve the diagnosis and treatment of this common disease. The new Rome IV criteria now supersede Rome III. In general, these minor amendments are unlikely to lead to substantial improvement in accuracy and use in routine clinical care.

Clinical criteria for functional constipation (FC) in elderly consist of $<3$ bowel movements per week, straining, the presence of lumpy or hard stools, the sensation of anorectal obstruction, the sensation of incomplete defecation, and manual maneuvers ${ }^{1}$. Almost all symptoms are subjective. This is so-called self-defined constipation. These criteria are significantly different from the criteria for obstructive constipation in children (1-18 years old), where the stool has a large diameter and emptying occurs with large volumes of feces ${ }^{2}$. It is obvious that the clinical criteria of FC in the elderly do not allow to put a pathophysiological diagnosis. If the organic causes of constipation are excluded (cancer, anal fissure, etc.) and the standard treatment has no effect, these symptoms serve for the initial selection of patients for following examination (digital rectal examination, anorectal manometry, balloon expulsion test, defecogaphy and colonic transit study) ${ }^{3}$. It is known, that the amount of obstructive constipation in the elderly does not increase, in comparison with the young, but the total number of patients with FC is almost 2 times greater. There is no unambiguous answer about the pathogenesis of this type of FC. Roque and Bouras subdivide FC on pelvic floor dysfunction and slow colonic transit. Abnormalities in pelvic floor dysfunction include failed relaxation of the pelvic floor, paradoxical contraction of the pelvic floor muscles, or the inability to produce the necessary propulsive forces needed in the rectum to expel the stool completely ${ }^{1}$. De Giorgio et al. describe other causes of FC: slow colonic transit, outlet obstruction: constipation by difficult or unsatisfactory expulsion of feces from rectum and constipation in IBS $^{3}$. Andromanakos et al consider that primary FC can be classified into normal transit constipation, slow transit constipation, and pelvic outlet obstruction ${ }^{4}$. The purpose of this study is to examine the X-ray symptoms of functional constipation in the elderly and to determine their origin.

\section{MATERIAL AND METHODS}

The barium enema, as described below, was performed to 37 patients aged 65 to 93 years with complaints of chronic constipation. Twenty-two patients were female, 10 males. This group does not include patients with tumors and rectal fissure. The control group consisted of 15 adolescents (11-17 years) without disturbance of the intestinal function. They had a barium enema to diagnose the cause of anemia, chronic abdominal pain and localization of the space occupation lesion. The results of this study were accepted as a norm. In addition to the standard barium enema, we applied a barium paste to the perineum near the anus or a radio contrast marker attached to the tip, which was located near the anus. On the lateral radiographs of the anorectal zone, the distance between the barium in the rectum and the contrast mark near the anus along the posterior contour of the tip was measured. This non-contrast zone forms an anal canal closed around the tip. Thus, the length of this distance is equal to the length of the functioning anal canal. The width of the rectum was measured in the widest part above the anal canal (Fig.1, A). In patients of the control group, a barium was introduced into the colon up to the reflux into the terminal ileum. In elderly patients, a contrast agent was administered in a volume of up to $300 \mathrm{ml}$, i.e up to the splenic flexure of the colon. First, because some patients could not retain more of the barium volume, secondly, as our studies have shown, the 

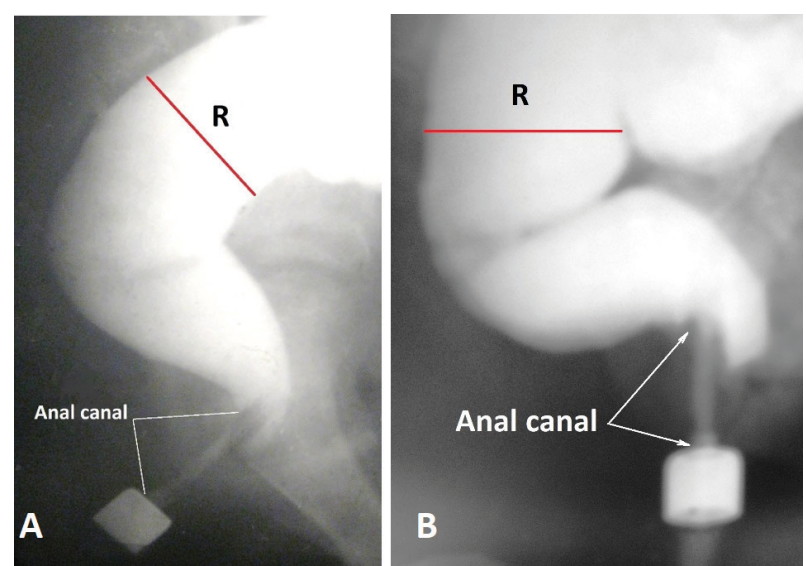

Figure 1. Lateral radiographs of the anorectal zone in patients of the control group. The rectum is bent forward resulting in a vertical and horizontal branch of the rectum can be distinguished. Between the rectum and the marker there is no contrast agent, since the anal canal contracted around the tip of the enema. B. As a result of relaxation of the internal anal sphincter, the barium penetrated into the upper part of the anal canal in front of the tip of the enema. The posterior wall of the anal canal at this level is pressed against the tip of the enema by a contracted puborectalis muscle. This is the X-ray equivalent of the anorectal inhibitory reflex

filling of the colon proximal to the splenic flexure does not change the parameters of the rectum and anal canal.

The radiographs were done after the administration of 100 , $200,300 \mathrm{ml}$ of barium. After that, the frontal abdominal radiograph was made. The true dimensions were calculated by multiplying the parameters measured on the roentgenogram by the projection increase factor, which under standard conditions is 0.72 . Statistical analysis was performed by the method of the Student's t-test. The level of significance was set as $\mathrm{P}<0.05$.

\section{RESULTS}

In the control group, the length of the anal canal along the posterior contour of the tip was within $3.1-3.7 \mathrm{~cm}(3.43 \pm$ $0.07 \mathrm{~cm}$ ) and was unchanged throughout the study. Period-

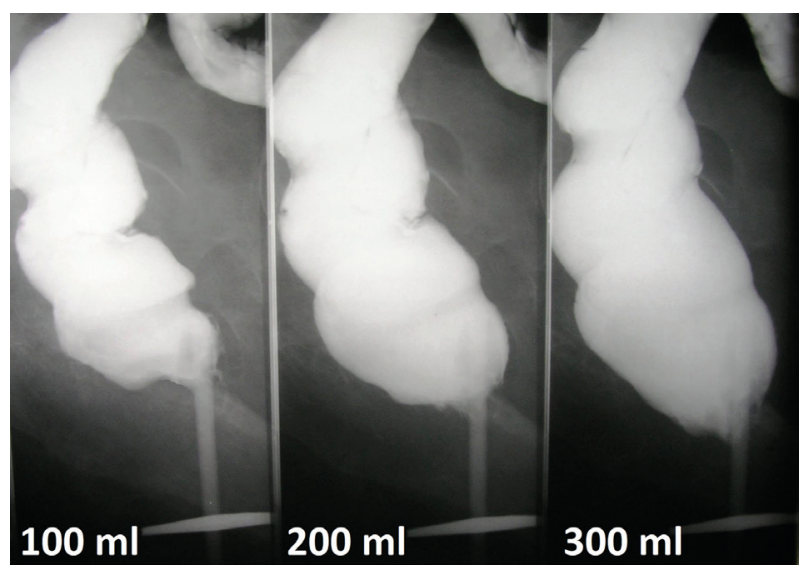

Figure 2. In a woman of 67 years during the barium enema produced three radiographs after the administration of 100, 200 and $300 \mathrm{ml}$ of contrast medium. The more barium was introduced, the shorter was the functioning anal canal. After the administration of $300 \mathrm{ml}$, the anal canal was reduced by $48 \%$ compared to the length after administration of $100 \mathrm{ml}$.

ically, the barium penetrated into the upper part of the anal canal in front of the enema tip, while the posterior wall of the anal canal at this level densely pressed against this tip (Fig. 1, B). After a few (7-12) seconds the barium was squeezed into the rectum and disappeared from the anal canal.

Only in $3(8 \%)$ patients the length of the anal canal was normal and did not change during the whole study. In the remaining observations, the anal canal was significantly shorter than in the control group (Table 1).

We noticed that in some cases the anal canal decreases during filling of the bowel with barium. The more barium is introduced, the shorter the anal canal becomes (Fig. 2). In other cases, the anal canal was twice shorter the minimum limit norm immediately after the onset of barium administration. They always had an expanded rectum. Therefore, in cases where $300 \mathrm{ml}$ of barium were administered to the colon, we grouped them according to the width of the rectum (Table 2).

In patients with a rectum width of more than $5 \mathrm{~cm}$, which

Table 1. Dependence of the rectal width and the anal canal length on the administration of the barium volumes.

\begin{tabular}{|c|c|c|c|c|c|}
\hline \multirow{2}{*}{ Parameters } & \multirow{2}{*}{ Control group } & \multicolumn{4}{|c|}{ Elderly patients (65-94 years) } \\
\cline { 3 - 6 } & & 1st X-ray & 2nd X-ray & 3rd X-ray & p \\
\hline \multirow{3}{*}{ The rectal width $(\mathrm{cm})$} & 15 & 37 & 37 & 23 & \\
& $3.6-4.6$ & $1.4-5.7$ & $2.5-6.7$ & $3.5-7.4$ & $\mathrm{p}^{1}-\mathrm{p}^{4}<0.01$ \\
\hline \multirow{3}{*}{ The anal canal length $(\mathrm{cm})$} & $3.95 \pm 0.07$ & $3.01 \pm 0.17$ & $4.23 \pm 0.18$ & $4.69 \pm 0.22$ & \\
\hline & 13 & 37 & 37 & 22 & $\mathrm{p}^{1}-\mathrm{p}^{2}>0.2$ \\
& $3.1-3.9$ & $2.0-5.1$ & $1.5-3.9$ & $1.5-3.7$ & $\mathrm{p}^{1}-\mathrm{p}^{3}<0.001$ \\
$\mathrm{p}^{1}-\mathrm{p}^{4}<0.001$ \\
\hline
\end{tabular}

Table 2. Dependence of the anal canal length on the width of the rectum.

\begin{tabular}{|c|c|c|c|c|}
\hline Parameters & & $\begin{array}{c}\text { The rectal width } \\
<5 \mathrm{~cm}\end{array}$ & $\begin{array}{c}\text { The rectal width } \\
>5 \mathrm{~cm}\end{array}$ & $\mathbf{p}$ \\
\hline The rectal width $(\mathrm{cm})$ & $\begin{array}{c}n \\
\text { fluctuation limits } \\
M \pm n\end{array}$ & $\begin{array}{c}17 \\
3.5-5.0 \\
4.16 \pm 0.11\end{array}$ & $\begin{array}{c}6 \\
5.2-7.4 \\
6.21 \pm 0.37\end{array}$ & $\begin{array}{c}\mathrm{p}^{1-2} \\
<0.001\end{array}$ \\
\hline \multirow[t]{2}{*}{ The anal canal length $(\mathrm{cm})$} & $\begin{array}{c}\mathrm{n} \\
\text { fluctuation limits } \\
\mathrm{M} \pm \mathrm{n}\end{array}$ & $\begin{array}{c}17 \\
1.5-3.7 \\
2.55 \pm 0.11 \\
\end{array}$ & $\begin{array}{c}6 \\
1.5-2.8 \\
1.97 \pm 0.18 \\
\end{array}$ & $\begin{array}{c}\mathrm{p}^{1-2} \\
<0.02\end{array}$ \\
\hline & & $\mathrm{p}^{1}$ & $\mathrm{p}^{2}$ & \\
\hline
\end{tabular}




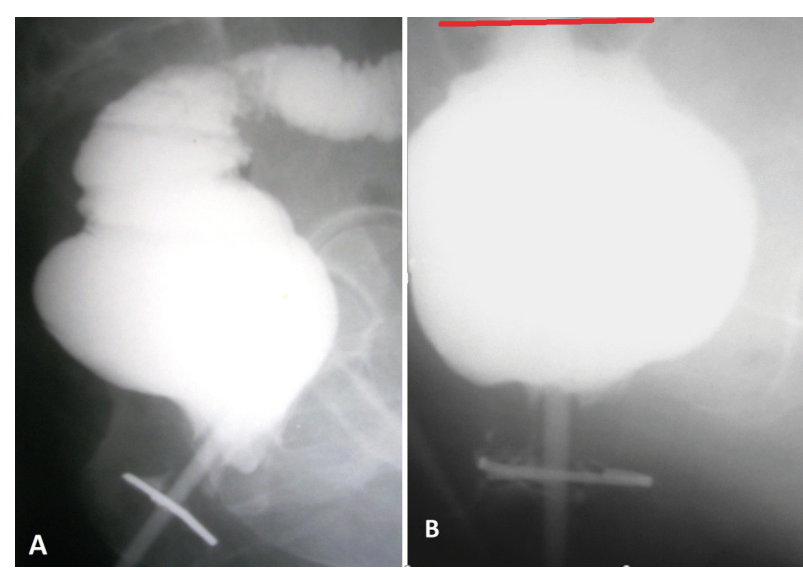

Figure 3. Lateral radiographs of the anorectal zone of two elderly patients with constipation, who have an expansion of the rectum. In both cases, there is no horizontal branch of the rectum. The anal canal is 2 times shorter than the minimum limit of normal. A. Sigmoid colon is narrow, irritated, which can be the result of prolonged use of bisacodyl. B. The width of the sigmoid colon is greatly expanded - megacolon. Sigmoid colon width - red line.

corresponds to the concept of obstructive constipation (Fig. 3 ), the length of the functioning anal canal was significantly less than in those where the width of the rectum was less than $5 \mathrm{~cm}$, i.e. within the limits of the norm.

Meanwhile, in patients with normal rectal width (up to 5 $\mathrm{cm}$ ), the length of the anal canal also was significantly less than in the control group ( $p<0.001)$. The shortening of the anal canal immediately after the introduction of the first portion of the barium is corresponds to the notion of a fixed descending perineum syndrome.

In 6 patients without rectal expansion on the frontal abdominal radiographs, signs of high colonic tone were found, which was the probable cause of slow transit constipation. Among them were 3 women with severe irritable bowel syndrome (Figure 4, A), two patients with several functional sphincters in the colon (Figure 4, B) and one man with diverticula of the sigmoid colon (Fig- 4, C).

\section{DISCUSSION}

The gap between the barium into the rectum and the marker near the anus is due to the contraction of the anal canal. In patients of the control group, its length is equal to the length of the anal canal measured by the manometric, ultrasound and MRI methods 5 .

In healthy individuals during the barium enema, the length of the anal canal, along the posterior contour of the enema tip remains unchanged. Periodically penetration of barium from the rectum into the upper part of the anal canal in front of the enema tip observed, which is accompanied by a decrease in anal pressure. This picture is due to the relaxation of the internal anal sphincter (IAS). At this time, the posterior wall of the anal canal is pressed against the tip of the enema by the contracted puborectalis muscle (PRM), which together with the external anal sphincter (EAS) prevents the leakage of barium. After 7-13 seconds, barium is squeezed back into the rectum, which is accompanied by the restoration of pressure to the basal level. This reaction of the anal canal to an increase in the rectal pressure is the radiographic equivalent of the inhibitory anorectal reflex ${ }^{5}$. In healthy people, the rectum on the lateral roentgenogram unfolds forward and is actually subdivided into vertical and horizontal branches.

Based on the analysis of clinical and radiological data, we found among 37 patients with FC the three homogeneous groups of patients. The first group consisted of $6(16 \%)$ patients with a rectal width more than $5 \mathrm{~cm}$. The second group comprised $25(68 \%)$ patients with normal rectum width, but with a short anal canal. The third group included $6(16 \%)$ patients with increased colonic tone. The width of the rectum and the length of the anal canal were within normal limits in 3 of them but in 3 cases the anal canal was shorter than normal.

In patients with megarectum (1st group), the anal canal was almost 2 times shorter than the minimum limit of norm and was always shorter than in patients in the 2 nd group. The shortening was detected immediately after the introduction of a small volume of barium. As shown by us earlier, the shortening of the functional part of the anal canal is due to the weakness of the pelvic floor muscles. Weak PRM cannot close up the upper part of the anal canal, and during a bowel movement a weak LAM cannot wide open the anal canal. Thus, the upper part of the anal canal is expanded and looks as a part of the rectum ${ }^{6}$.

This picture corresponds to concept of a fixed form of the descending perineal syndrome ${ }^{7}$. In the first group there was always no horizontal branch of the rectum. Based on these six observations, we came to the conclusions that the reason of constipation in them is an outlet obstruction that arose either in childhood or in the late period as a result of anal stenosis or fissures. Stool retention in the rectum led to the development of a megarectum. Wide feces under the influence of a strong peristaltic wave stretched the upper part of the anal canal, in which the stool masses begin to accumulate. In 25 patients of the second group the width of the rectum was within normal limits. They did not suffer from abdominal pain and in the frontal radiograph the large intestine had normal size and shape. They had two branches of the rec-
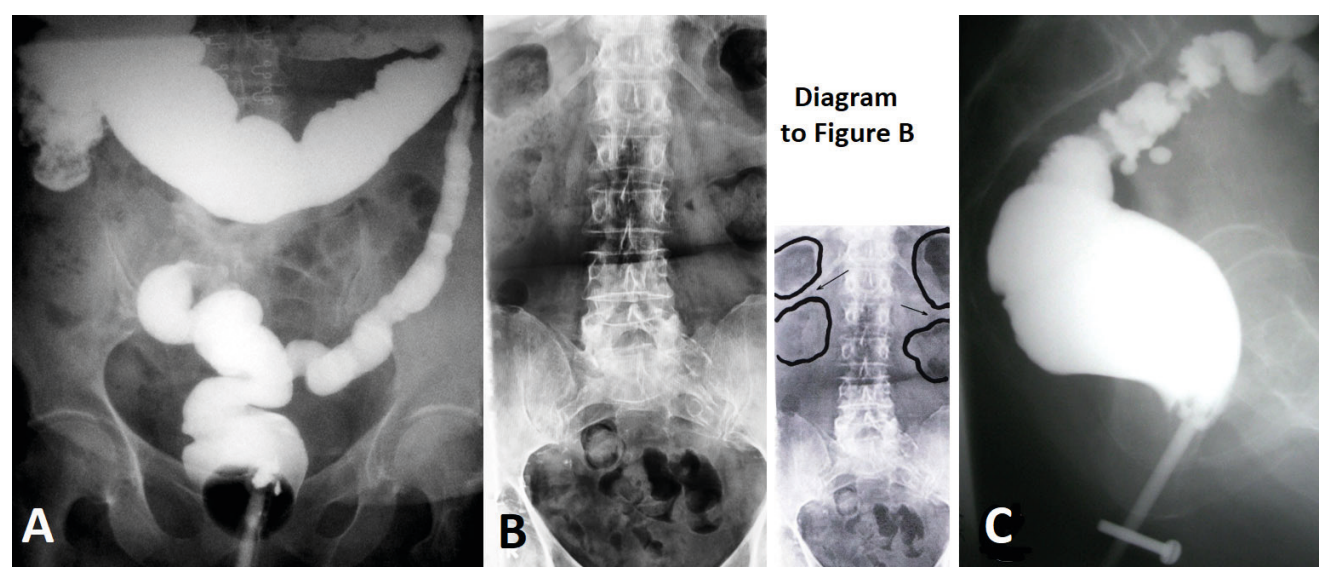

Figure 4. Radiographs of patients with increased tone of different segments of the colon. A. In a patient with irritable bowel syndrome, the left half of the colon is sharply narrowed, without haustration. B. The arrows show functional sphincters in the form of colon contraction, between adjacent chambers containing gas and feces. The rectum is empty. C. High tone of the sigmoid colon with diverticula. The size of the rectum and anal canal is within normal limits. 

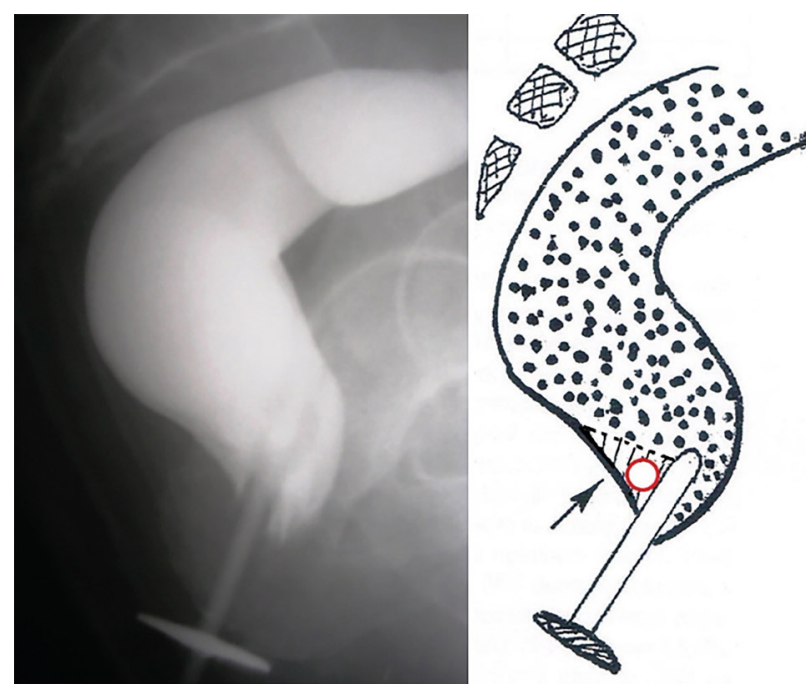

Figure 5. Lateral radiograph of the anorectal zone of an elderly man with complaints of constipation and incomplete emptying. The dynamic descending perineum syndrome with penetration of barium into the upper part of the anal canal behind the tip of the enema is determined (arrow in the diagram). The red ring indicates a fecal bolus that has penetrated the anal canal.

tum: vertical and horizontal. The anal canal was shortened during the introduction of the contrast medium, which corresponds to the dynamic form of the descending perineum syndrome ${ }^{7}$.

Consequently, in these patients, the pelvic floor muscle weakness was less pronounced than in patients of first group with outlet obstruction. It is caused by age-related changes in the muscles of the pelvic floor. The normal width of the rectum indicates that the anal canal did not interfere with the promoting of the stool. We assumed that in these cases there is a so-called self-determined form of constipation, which is characterized by patients' complaints about the difficulty of defecation and a feeling of incomplete emptying. The mechanism of occurrence of these sensations can be explained by the penetration of small fecal boluses into the upper part of the anal canal due to the weakness of the PRM. Irritation of sensory elements in this part of the anal canal by fecal bolus causes a need for defecation, but a small amount of bolus is unable to create a threshold volume of defecation. Because of this, patients are forced to use a finger maneuver, i.e. removal of feces with a finger through the vagina or anus. The rest of small fecal lumps in this part of the anal canal provokes a feeling of incompletely emptying (Fig.5).

In 6 patients of the third group, FC was combined with abdominal pain. The width of the rectum and the length of the anal canal were within normal limits in 3 of the patients, and in the remaining 3 patients with irritable bowel syndrome the rectum was narrow. On the frontal radiographs the signs of an increased tone of the colon of different nature were found, which inevitably caused the slow transit through the colon.

One of the reasons for the high tone of the colon is irritable bowel syndrome (IBS). Some researchers exclude IBS from the analysis of $\mathrm{FC}^{8}$. We consider necessary to include it in a differential series for two reasons. First, because there are no clear clinical criteria for diagnosis of IBS Secondly, as we showed earlier, obstructive constipation, as a form of chronic obstruction, can cause the development of nonspecific inflammation of the colon and an increase its tone. In such cases, chronic constipation may be accompanied by pain in the abdomen (see Fig. 3.A) and differential diagnosis of FC and IBS is not possible. The main pathognomonic symptoms of IBS are the increased sensitivity of the colon to intestinal irritation (pressure) ${ }^{9}$. It is necessary to distinguish delayed transit through the colon, as a result, of an increase in the tone of its different segments, which is the cause of FC, from delayed transit, which in obstructive constipation is observed as a symptom. Prolonged accumulation of large feces in the rectum and left colon invariably causes a slowing of the movement of feces.

In the process of ontogenesis, functional sphincters appear in the colon. A total of 11 functional sphincters are described. The most extensively studied are colosigmoid sphincter "(CSS) and rectosigmoid sphincter (RSS). Between the descending and sigmoid colon there is a physiological CSS by a length of $2.1 \pm 0.9 \mathrm{~cm}$. In this zone, the pressure was significantly higher than in the adjacent segments. After a quick inflation of the balloon of large diameter in the descending colon, the decrease of pressure in the CSS was noted. After inflating the same balloon in the sigmoid colon, the pressure in the CSS increases. On the other hand, inflation with a small balloon did not affects the tone of this sphincter. It is clear that the CSS is involved in a passage of large intestinal contents. CSS retains the feces until it reaches a certain volume ${ }^{10}$. Between the rectum and sigmoid is located the intestinal segment of $2.8 \pm 0.9 \mathrm{~cm}$ length with the layer of circular muscle thickened in comparison to the segments above and below it $^{11}$. While the pressure in the sigmoid colon increases, this functional RSS relaxes. In response to increased pressure in the rectum, its tonus rises ${ }^{12}$. Different reactions of RSS to different volume of the balloon were found. After a quick inflation of the balloon in the sigmoid with $52.1 \pm 3.6 \mathrm{ml}$ of liquid, the tone of the RSS increased but the rectal pressure did not change. After the rapid inflation into the balloon of $86 \pm 4.1 \mathrm{ml}$, the RSS relaxed and the balloon was dispelled to the rectum. It was accompanied by an increase of the rectal pressure, and the balloon was expelled ${ }^{13-14}$. On the roentgenogram of the spine (Fig.4.B), at least 4 oval chambers containing gas and feces are seen that are separated from each other by contracted functional sphincters. They are opened and passed feces only when a large volume of feces gathers in the chambers above the sphincters. This dramatically slows the passage of feces across the colon, which corresponds to the concept of slow transit constipation.

High pressure in the sigmoid colon can be so strong that diverticula are formed. It is always accompanied by chronic constipation and abdominal pain. However, as can be seen in Fig. 4.C, the rectum and anal canal are of normal size and shape and do not cause chronic constipation. This is also one of the forms of slow transit constipation in the elderly.

\section{CONCLUSION}

We found 3 types of FC in the elderly. In the structure of FC are dominated by cases of self-defined constipation (68\%), where there is no mechanical obstruction for defecation. The reason for the sense of constipation and incomplete emptying is due to the age-related weakness of the pelvic floor muscles. In $16 \%$ of patients, obstructive constipation with a megarectum and a fixed descending perineum syndrome was detected. This pathology originated in childhood or after anal fissures, and childbirth. In $16 \%$ of patients, the cause of constipation was a high tone of the colon, what can be the result of an irritable bowel syndrome, the formation of functional sphincters in the colon and diverticula of the sigmoid colon. An increase in the tone of the colon causes a slow transit constipation. 


\section{REFERENCES}

1. Maria Vazquez Roque and Ernest P Bouras. Epidemiology and management of chronic constipation in elderly patients. Clin Interv Aging. 2015; 10: 919-930. Published online 2015 Jun 2. doi: $10.2147 /$ CIA.S54304

2. Elvira Ingrid Levy, Roel Lemmens, Yvan Vandenplas, and Thierry Devreker. Functional constipation in children: challenges and solutions. Pediatric Health Med Ther. 2017; 8: 19-27. Published online 2017 Mar 9. doi: 10.2147/PHMT. S110940

3. De Giorgio R, Ruggeri E, Stanghellini V et al. Chronic constipation in the elderly: a primer for the gastroenterologist. BMC Gastroenterol. 2015 Oct 14;15:130. doi: 10.1186/s12876-0150366-3.

4. Andromanakos NP, Pinis SI, Kostakis AI. Chronic severe constipation: current pathophysiological aspects, new diagnostic approaches, and therapeutic options. Eur J Gastroenterol Hepatol. 2015; 27(3):204-14. doi: 10.1097/MEG.0000000000000288.

5. Levin MD. The role of the external anal sphincter in the physiology of the pelvic floor. Pelviperineolohy. 2017; 36(4):108112 .

6. Levin MD. Pathophysiology and diagnosis of descending perineum syndrome in children. Pelviperineology. 2018;37(2):52-54

7. Baek HN, Hwang YH, Jung YH. Clinical Significance of Perineal Descent in Pelvic Outlet Obstruction Diagnosed by using Defecography. J Korean Soc Coloproctol. 2010;26(6):395401. doi: 10.3393/jksc.2010.26.6.395. Epub 2010 Dec 31.

8. Bouras EP, Tangalos EG. Chronic constipation in the elderly. Gastroenterol Clin North Am. 2009;38(3):463-80. doi: 10.1016/j.gtc.2009.06.001.

9. Ritchie J . Pain from distension of the pelvic colon by inflating a balloon in the irritable colon syndrome. Gut. 1973;14(2):125-32.

10. Shafik A. Effect of rectal distension on the small intestine with evidence of a recto-enteric reflex. Hepatogastroenterology.2000; 47(34):1030-3
11. Shafik A, Shafik AA, el-Sibai O, Ahmed I. Colosigmoid junction: a study of its functional activity with identification of a physiologic sphincter and involvement in reflex actions. J Invest Surg. 2003;16(1):29-34.

12. Shafik A, Assad S, Doss S. Identification of a sphincter at the sigmoidorectal canal in humans: histomorphologic and morphometric studies.Clin Anat. 2003 Mar;16(2):138-43.

13. Shafik A. The hypertonic rectosigmoid junction: description of a new clinicopathologic entity causing constipation. Surg Laparosc Endosc. 1997;7(2):116-20.

14. Shafik A, Shafik AA, El Sibai O, Ahmed I, Mostafa RM. Role of the rectosigmoidal junction in fecal continence: concept of the primary continent mechanism. Arch Surg. 2006;141(1):23-6.

\section{NOTES}

For the first time functional constipation in the elderly is assessed from the point of view of the anorectal zone function using mathematical analysis.

\section{DISCLOSURES}

There was no conflict of interest, informed patient consent was obtained, the study was approved by the local ethical committee and the author of the publication did not receive any financial support by any grant/research sponsor.

Correspondence to:

Michael Levin MD, Dsc. Radiologist

Amnon veTavar 1/2 - Netanya 42202 - Israel

Tel.: 972-538281393

E-mail: nivel70@hotmail.com

F-michael.levin@dorot.health.gov.il

https://orcid.org/0000-0001-7830-1944

https://www.anorectalmalformations.com

\section{Multidisciplinary UroGyneProcto Editorial Comment}

To improve the integration among the three segments of the pelvic floor, some of the articles published in Pelviperineology are commented on by Urologists, Gynecologists, Proctologists/Colo Rectal Surgeons or other Specialists, with their critical opinion and a teaching purpose. Differences, similarities and possible relationships between the data presented and what is known in the three fields of competence are stressed, or the absence of any analogy is indicated. The discussion is not a peer review, it concerns concepts, ideas, theories, not the methodology of the presentation.

Uro... It is well known that human defecation is a complex physiologic mechanism that involves integrated and coordinated sensorimotor functions orchestrated by central, spinal and peripheral neural activities. In this way when the rectum is filled with feces the pressure within it is increased allowing the passage of the fecal material through the anal canal which has further increased by muscles of pelvic floor helping to pull the anal canal walls apart and causing a further filling of the anal canal by feces that can be expelled due to relaxation of internal and external spinchters.

This coordinated neuromuscular activity is analogous also for what concerns filling and bladder emptying phase, since the innervation of the bladder and of sphincteric mechanism is similar to the rectum and of the anal canal one.

A close functional relationship between urinary retention and constipation in the elderly is therefore evident. In fact in this age group it is very probable the presence of a detrusor underactivity like an equally disabling condition to produce the necessary propulsive forces needed in the rectum to expel the stool completely. Most likely the cause, from an uro- logical point of view, is due to a transformation of the detrusor muscle tissue into collagen tissue due to a prolonged obstruction over time of the bladder neck caused by prostatic hypertrophy or a large vaginal prolapse ${ }^{1}$. Much more rarely, instead, the urinary retention in the elderly is due to a failed relaxation of the pelvic floor as this pathological condition is typical of young subjects affected by chronic pelvic pain.

\section{REFERENCE}

1. Bellucci CHS, Ribeiro WO, Emerly TS et al. Increased detrusor collagen is associated with detrusor overactivity and decrease bladder and compliance in men with benign prostatic obstruction. Prostate Int 2017; 5: 70-74

Correspondence to:

SALVATORE SIRACUSANO

Professor of Urology

University of Verona, Italy

Email: salvatore.siracusano@univr.it 